\title{
STRUCTURE OF THE CIRCUMSTELLAR ENVELOPE OF
}

\author{
$\psi$ PERSEI AT LARGE RADII
}

\author{
S.M. DOUGHERTY \\ John Moores University, Liverpool, England \\ and \\ A.R. TAYLOR \\ University of Calgary, Calgary, Canada
}

\begin{abstract}
The radiative transfer equation is solved for a generalised disc model of the circumstellar envelope around $\psi$ Persei to investigate the nature of the long wavelength turndown in the continuum spectrum of Be stars. The flux density and source dimensions at $15 \mathrm{GHz}$ rule out truncation of the circumstellar envelope around $\psi$ Persei as the cause of the turndown.
\end{abstract}

The excess thermal continuum spectra of radio detected Be stars exhibit a turndown longward of the far-IR wavelength regime (Taylor et al., 1990). This feature has not been observed in the continuum spectra of other hot stars. Several mechanisms have been proposed to account for this turndown, including truncation of the circumstellar envelope.

The far-IR region of Be star spectra have a power-law form which can be interpreted as arising from a plasma with a radial density distribution of the form $\rho \propto r^{-\beta}$, where $\beta$ is the density index. For such a circumstellar plasma the optical depth along a line of sight $z$ is given by

$$
\tau_{\nu}(z) \propto \int_{\zeta}^{\xi} r^{-2 \beta} d z
$$

where $\zeta$ and $\xi$ are limits of integration determined by the geometry of the circumstellar plasma envelope, namely the radius of the disc, the inclination of the rotation axis of the star to the line of sight $(i)$, and the half-opening angle of the disc $(\theta)$. Constraints on the model parameters $\theta$ and $i$ can be determined from this model in conjunction with the observed minimum aspect ratio of 1.6 for the $15 \mathrm{GHz}$ emission region (Dougherty and Taylor, 1992). In addition, the presence of shell lines e.g. Oegerle and Polidan (1984) implies $\theta+i \geq 90^{\circ}$. It is found that $i>67^{\circ}$ for $\psi$ Persei (Dougherty, 1993).

The best fit model (Figure 1) was determined by using a weighted minimum $\chi^{2}$. The most striking feature of the fit is that to account for the spectral turndown requires that the disc model is truncated at a radius of $560 R_{\odot}$. This is much smaller than the observed lower limit to the radius of the emitting region of $1850 R_{\odot}$ (Dougherty and Taylor, 1992). A disc model with a radius of $1850 R_{\odot}$ gives radio fluxes that are too high. Clearly, a truncated disc model cannot account for both the radio flux densities and the 


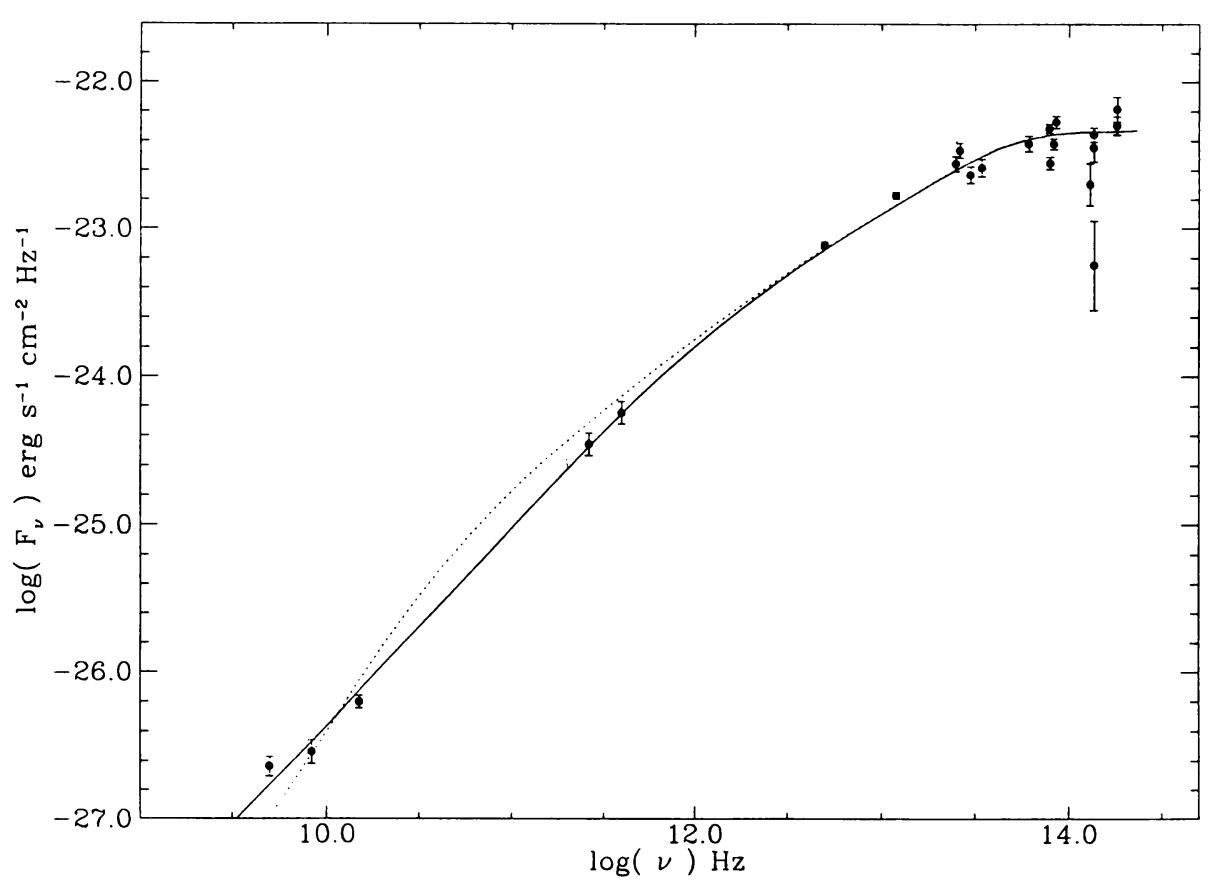

Fig. 1. Optimal fit of the truncated (dashed) and double density index (solid) models to the observed excess spectrum of $\psi$ Persei. Clearly, the truncated model gives an unsatisfactory fit to the radio and millimeter observations, whereas the solid line represents an excellent fit at all wavelengths.

observed size of the radio emitting region. This rules out disc truncation as the mechanism causing the long wavelength turndown observed in $\psi$ Persei.

The spectrum is best fit by an infinite disc model that has a change in the density index at a radius of $166 \pm 57 R_{\odot}$ (Figure 1 ). Clearly, a change in the density index at $\sim 170 R_{\odot}$ can account for both the radius of the $15 \mathrm{GHz}$ emitting region and the long wavelength turndown in the excess spectrum. Re-combination of electrons and ions, a disc opening angle that is a function of radius, and acceleration at large distances from the underlying star are all mechanisms that could give rise to such a change in density index (Taylor et al., 1990; Waters et al., 1991)

\section{References}

Dougherty, S.M., and Taylor, A.R. 1992, Nature, 359, 808

Dougherty, S.M. 1993, PhD thesis, University of Calgary.

Oegerle, W.R., and Polidan, R.S. 1984, Astrophysical Journal, 285, 648

Taylor, A.R., Waters, L.B.F.M., Bjorkman, K.S., Dougherty, S.M. 1990, Astronomy and Astrophysics, 231, 453.

Waters, L.B.F.M., van der Veen, W.E.C.J., Taylor, A.R., Marlborough, J.M., Dougherty, S.M. 1991, Astronomy and Astrophysics, 244, 120 